\title{
Spatial and temporal dynamics of cortical networks engaged in memory encoding and retrieval
}

\author{
Brian T. Miller* and Mark D'Esposito \\ Helen Wills Neuroscience Institute, University of California, Berkeley, CA, USA
}

\section{Edited by:}

Stefano F. Cappa, Vita-Salute San

Raffaele University, Italy

\section{Reviewed by:}

Hidenao Fukuyama, Kyoto

University, Japan

Kirsten G. Volz, Werner Reichardt

Centre for Integrative Neuroscience,

Germany

\section{${ }^{*}$ Correspondence:}

Brian T. Miller, Helen Wills

Neuroscience Institute, University

of California, 132 Barker Hall,

MC\#3190, Berkeley,

CA 94720-3190, USA.

e-mail:bmiller@gmail.com
Memory operations such as encoding and retrieval require the coordinated interplay of cortical regions with distinct functional contributions. The mechanistic nature of these interactions, however, remains unspecified. During the performance of a face memory task during fMRI scanning, we measured the magnitude (a measure of the strength of coupling between areas) and phase (a measure of the relative timing across areas) of coherence between regions of interest and the rest of the brain. The fusiform face area (FFA) showed robust coherence with a distributed network of subregions in the prefrontal cortex (PFC), posterior parietal cortex (PPC), precuneus, and hippocampus across both memory operations. While these findings reveal significant overlap in the cortical networks underlying mnemonic encoding and retrieval, coherence phase analyses revealed context-dependent differences in cortical dynamics. During both encoding and retrieval, PFC and PPC exhibited earlier activity than in the FFA and hippocampus. Also, during retrieval, PFC activity preceded PPC activity. These findings are consistent with prior physiology studies suggesting an early contribution of PFC and PPC in mnemonic control. Together, these findings contribute to the growing literature exploring the spatio-temporal dynamics of basic memory operations.

Keywords: functional connectivity, effective connectivity, fMRI, episodic memory, prefrontal cortex, posterior parietal cortex, hippocampus

\section{INTRODUCTION}

Successful formation and retrieval of visual memory representations fundamentally rely on the integration of bottom-up perceptual and maintenance/storage operations with top-down mnemonic control processes (Ranganath and D'Esposito, 2005). The neural dynamics of these interactions lay at the very foundation of the human ability to capture relevant sensory information and transform it into high fidelity representations that can be used in the short and long-term service of behavior. Recent evidence has shown that the active, temporary maintenance of face representations in the absence of bottom-up sensory input [e.g., working memory (WM)] is mediated by coupling between frontal regions and the fusiform face area (FFA) (Gazzaley et al., 2005). Other studies have found similar coupling between prefrontal cortex (PFC) and both extrastriate cortex (Summerfield et al., 2006) and the medial temporal lobe (Ranganath et al., 2005) during the formation and retrieval of visual long-term memory (LTM) representations.

Other multimodal regions thought to initiate control signals that modify perceptual processes are subregions in posterior parietal cortex (PPC). While previously linked with visuospatial contributions to the perception of memoranda rather than mnemonic processing per-se (for a review of the link between attention and memory, see Chun and Turk-Browne, 2007), recent evidence has linked parietal activity to episodic memory. For example, during encoding lateral PPC exhibits increased activity for subsequently remembered items (for a review, see: Uncapher and Wagner, 2009) and during mnemonic retrieval (Buchsbaum et al., 2011). PPC also exhibits increased activity reflecting "retrieval success" (Konishi et al., 2000), recollection versus familiarity (Dobbins et al., 2003; Wheeler and Buckner, 2004), and attempts to retrieve source information (Cabeza et al., 2003). Further evidence for a role of the PPC in episodic memory comes from anatomical evidence showing reciprocal connectivity between the PPC and both lateral temporal regions (Takahashi et al., 2007) and the hippocampus (Suzuki and Amaral, 1994). Moreover, the PPC regions engaged during episodic memory retrieval are distinct from those engaged during both exogenous and endogenous attention (Hutchinson et al., 2009). Though these findings point to a specific role of PPC in episodic memory-potentially through interactions with sensory and/or MTL regions- the specific functional role of the PPC as well as the nature of its network interactions remain underspecified.

Human imaging studies, then, implicate coupling between multimodal PFC and PPC control regions and both perceptual and MTL regions as a key mechanism responsible for mnemonic function. These functional and anatomical connectivity studies, however, have not uncovered the dynamics of the interactions and directionality of signaling between these regions. Such information is vital for understanding encoding and retrieval operations in order to characterize the specifics of the integration processes emanating from bottom-up sensory versus top-down brain regions. This integration is likely a mechanism by which representations are strengthened at encoding (leading to consolidation of the memory trace) and then flexibly accessed at retrieval. 
Despite a wealth of suggestive empirical evidence and theoretical models implicating the parietal (Knudsen, 2007) and PFC (Mesulam, 1998; Shimamura, 2000; Miller and Cohen, 2001) in top-down control of memory and perception, direct evidence for these top-down signals to visual regions remains limited due to methodological challenges in detecting causal influences between brain areas (Miller and D'Esposito, 2005). As a result, important information about the systems-level mechanisms underlying memory operations remains elusive.

One approach toward characterizing these direct influences is to track regional timing information. Latency measures reflect the order of physiological activity across regions known to be functionally coupled and can index the cascade of signaling over time. Single-unit neurophysiology provides millisecond level temporal measurements with spatial precision but is limited by technical challenges in simultaneously recording from multiple brain regions. However, recently simultaneous recording from neurons in two brain regions during the same behavioral task has been achieved (e.g., Buschmman and Miller, 2007; Antzoulatos and Miller, 2011).

Functional MRI, on the other hand, by virtue of its wholebrain sampling has been an invaluable tool for delineating regions within these functional networks, but has contributed less information about the dynamic interplay between regions in memory function. This is due to the fact that only a few studies have employed multivariate analyses of fMRI data capable of measuring both functional and effective connectivity. One of the analytic tools used to explore these interactions is Dynamic Causal Modeling (DCM) - a statistical method that uses a Bayesian framework with a number of features including a biophysical forward model to link a neurophsyiological model with features of the measured BOLD fMRI or electrophysiological signals. Work with DCM has delineated a range of top-down interactions in attentional control (Wang et al., 2010), object recognition (Kveraga et al., 2007), mental imagery (Mechelli et al., 2004) as well as a contribution of top-down effects on late stage perceptual evoked electrophysiology signals (Garrido et al., 2007).

In this experiment we use another analytic tool, multivariate coherence analysis (Sun et al., 2004), to explore the spatiotemporal dynamics of episodic encoding and retrieval. While fMRI signals are a hemodynamic correlate of neural activity, evaluating changes in the temporal properties of BOLD fMRI responses across conditions and changing temporal relationships across regions offers evidence about the changing interactions within neural networks. We use coherence magnitude first as a means to assess functional connectivity - that is, the statistical dependency or correlation between remote neurophysiological time-series. We do so in a manner that is insensitive to regional variations in the hemodynamic response function (HRF). We then use coherence phase measures to complement existing neurophysiology literature and permit inferences about the directionality (e.g., effective connectivity) of these functional connections. Although these analyses cannot directly measure neural dynamics, we assess condition-dependent changes in the temporal nature of the underlying responses by examining changes during encoding and retrieval in the relative phase dynamics of the underlying networks.
In a face memory task, participants engaged in encoding and retrieval operations (i.e., old/new recognition decisions) across blocks of trials in the presence of identical bottom-up sensory input (i.e., single face stimuli). By measuring the magnitude of coherence across the brain relative to the the FFA, we identified highly overlapping networks across encoding and retrieval conditions. Coherence phase estimates provided further information about the temporal dynamics of these interactions addressing the role of top-down control signals in modulating bottom-up signals in the service of memory. These findings highlight the growing role of multivariate techniques in characterizing systems-level mechanisms of cortical networks.

\section{MATERIALS AND METHODS PARTICIPANTS}

The participants in this study were 15 undergraduates from the University of California at Berkeley (ages 19-27, $M=21.9$, nine female). All participants were right handed with normal or corrected-to-normal vision and none reported any history of neurological or psychiatric problems. Each participant gave informed written consent prior to being tested and received monetary compensation upon completion of the study. The following experimental procedure was conducted in compliance with the Committee for the Protection of Human Subjects at the University of California, Berkeley.

\section{BEHAVIORAL TASKS}

Two tasks were performed during fMRI scanning. Prior to the main experimental task, participants performed a one-back working memory task comprised of face and scene stimuli blocks. Data from this experimental run was utilized to localize FFA voxels to serve as a functionally defined seed region of interest to pass on to second stage coherence analyses of the main memory task (see statistical methods). Participants viewed 16 second blocks of 20 face or 20 scene stimuli presented for $300 \mathrm{~ms}$ each with a $500 \mathrm{~ms}$ ITI. To ensure that participants were viewing the stimuli, they were instructed to make a button-press with their right index finger any time that an image matched the image immediately preceding it. There were seven blocks of each category of stimuli and the localizer session lasted 5 min $45 \mathrm{~s}$.

Following this session, participants performed four experimental runs of a memory task in which they encoded and made recognition decisions about single face stimuli. A set of 360 grayscale photographs of human faces with neutral expressions was assembled. Several steps were taken to promote the use of face recognition, rather than recognition of extraneous features (e.g., hair, clothing). Image processing was carried out using Adobe Photoshop (version 6.0). All external features, such as hair, ears, and the background of the photograph, were cropped from the picture and replaced by a gray background. The boundary between the face and the background was then blurred by using the Photoshop "smudge" tool. All images were $174 \times 232$ pixels with a resolution of 72 DPI.

During the main experimental session, participants performed interleaved sessions of "Encoding" and "Retrieval" trials structured in independent experimental runs. During encoding runs, participants were presented with single-face stimuli on the screen 
for $2 \mathrm{~s}$ and were instructed to memorize the faces to the best of their ability in anticipation of a future memory test. Faces were presented throughout the six min run and separated by a jittered inter-trial interval of $4 \mathrm{~s}$ (50\% of the trials), $6 \mathrm{~s}$ (25\% of the trials), and $8 \mathrm{~s}$ (25\% of the trials). In order to balance motor demands across the two run types, participants were also instructed to make a button-press with their index finger upon viewing the face while they simultaneously attempted to memorize the face stimulus. Following the encoding run, participants took a 2 min break. Following the break, a retrieval run began in which participants once again saw individual faces on the screen for $2 \mathrm{~s}$ in an identically jittered manner. In retrieval trials, participants were instructed to make a recognition decision to each stimulus indicating whether it was in the previous encoding run or was a novel face. They indicated their decision with a button press with their index finger ("old") or their middle finger ("novel") of their right hand. Importantly, across encoding and retrieval runs, the nature of the bottomup visual input (i.e., a single face stimulus) was held constant and the only difference across blocks was the nature of the mnemonic operations performed when the faces were present. In all, there were 40 face stimuli in each block and each run lasted $6.5 \mathrm{~min}$.

\section{MRI ACQUISITION AND PRE-PROCESSING}

Functional images were acquired from a Varian INOVA 4 Tesla scanner equipped with a transverse electromagnetic (TEM) sendand-receive radio frequency (RF) head coil. Functional images were collected using a gradient echoplanar sequence $(\mathrm{TR}=2 \mathrm{~s}$, $\mathrm{TE}=28 \mathrm{~ms}$, matrix size $=64 \times 64, \mathrm{FOV}=22.4 \mathrm{~cm}$ ) sensitive to BOLD contrast. Each functional volume consisted of $18 \times 5 \mathrm{~mm}$ thick axial slices with $0.5 \mathrm{~mm}$ gap between each slice, providing whole brain coverage except for portions of the inferior cerebellum and the most superior extent of the parietal lobe. For each scan, $10 \mathrm{~s}$ of gradient and RF pulses preceded data acquisition to allow steady-state tissue magnetization. Two T1-weighted anatomical scans were also acquired. In the first, anatomical images coplanar with the EPI data were collected using a gradient-echo multislice (GEMS) sequence $(\mathrm{TR}=200 \mathrm{~ms}, \mathrm{TE}=$ $5 \mathrm{~ms}, \mathrm{FOV}=22.4 \mathrm{~cm}^{2}$, matrix size $=256 \times 256$, in-plane resolution $=0.875 \times 0.875 \mathrm{~mm}$ ). These images were used in later analyses to determine individual-specific regions of interest as well as to anatomically localize functional activations. In the second, high-resolution anatomical data were acquired with an MP-FLASH 3-D sequence $(\mathrm{TR}=9 \mathrm{~ms}, \mathrm{TE}=5 \mathrm{~ms}, \mathrm{FOV}=$ $22.4 \times 22.4 \times 19.8 \mathrm{~cm}$, matrix size $=256 \times 256 \times 128$, resolution $=0.875 \times 0.875 \times 1.54 \mathrm{~mm})$ to aid in spatial normalization (see below).

Following acquisition, MRI data were converted to ANALYSE format. Data were corrected for between-slice timing differences using a sinc interpolation method and were interpolated to $1 \mathrm{~s}$ temporal resolution (half of the total repetition time) by combining each shot of half $k$ space with the bilinear interpolation of the two flanking shots. Subsequent pre-processing and statistical analysis were performed using SPM2 software http://www. fil.ion.ucl.ac.uk) run under Matlab 6.5 (www.mathworks.com). Functional data were then realigned to the first volume acquired to account for intra- and inter-session movement. Following co-registration to the in-plane GEMS, the high resolution MPFLASH was normalized to a T1 template using a 12-paramater affine transformation along with a non-linear transformation using cosine functions. These normalization parameters were then applied to the functional images to transform each volume into standardized MNI space. These images were then spatially smoothed with an $8 \mathrm{~mm}$ FWHM gaussian kernel.

\section{STATISTICAL METHODS \\ Whole-brain univariate analysis}

A standard univariate analysis was conducted under the assumptions of the general linear model (GLM) for two purposes: (1) To identify cortical regions commonly or selectively engaged in memory encoding and retrieval operations in the presence of identical bottom-up sensory input. (2) To isolate a subset of these voxel clusters to pass on to a second-stage coherence analysis aimed at delineating the spatio-temporal dynamics of these networks.

Each event in the encoding and retrieval runs was modeled as a $2 \mathrm{~s}$ epoch and convolved with a canonical HRF. Eventrelated effects were estimated using a subject-specific fixed-effects model and linear contrasts were computed to isolate voxels significantly activated in encoding vs. baseline, retrieval vs. baseline, as well as a linear contrast of the two memory conditions. These subject-specific estimates were then entered into a second-level random effects analysis using a one-sample $t$-test against a contrast value of zero at each voxel. Task-common maps to guide seed localization for the subsequent coherence analyses were identified by taking a conjunction of encoding and retrieval group maps to determine voxels statistically significant in both conditions ( $p<0.001$, uncorrected). Task-selective maps to guide localization of operation-specific seeds for coherence analysis were identified as voxels significantly different across conditions in the linear contrast map ( $p<0.001$, uncorrected).

\section{Coherence analysis}

To obtain measures of functional and effective connectivity during encoding and retrieval, we performed a seed-based coherence analysis to measure the strength and phase delay of coherence between seed voxels of interest and the rest of the brain.

The magnitude of coherence is a measure of how well one signal can be represented as a linear transform of another. Coherence magnitude between time series $x$ and $y$ is defined by the equation:

$$
\operatorname{Coh}_{s y}(\lambda)=\left|R_{x y}(\lambda)\right|^{2}=\left[\left|f_{x y}(\lambda)\right|^{2}\right] /\left[f_{x x}(\lambda) f_{y y}(\lambda)\right]
$$

where $f_{x y}(\lambda)$ is the cross-spectrum of $x$ and $y$ and $f_{x x}(\lambda)$ and $f_{y y}(\lambda)$ are the respective power spectra of $x$ and $y$. Coherence magnitudes range from 0 to 1 , where 1 indicates that one time series can perfectly predict the other in a linear fashion.

A major strength of coherence measures in the context of fMRI BOLD signal is its invariance to differences in the shape of the HRF across regions and across individuals. Unlike pure correlation, the coherence between two signals with similar underlying neural patterns will be high even if the baseline HRFs across the regions have a systematic phase lags. In fact, a primary advantage 
of coherence is that systematic phase delays between two signals can provide reliable information about the temporal relationship of the underlying neurophysiological activity between regions (Sun et al., 2005; for a discussion of hemodynamic versus physiological time delays see discussion below). Assuming similar HRFs across regions, then, coherence is in a unique position to provide latency measures that can shed light on the temporal cascade of computations across the brain. Sun et al. (2005) validated this phase delay analysis method with fMRI by replicating known physiological measures in the context of a bimanual motor task.

The phase spectrum, $\phi$ is defined as

$$
\tau(\lambda)=\arg \left\{R_{x y}(\lambda)\right\}=\arg \left\{f_{x y}(\lambda)\right\}
$$

where $\arg \{\}$ represents the argument. The group delay, $\tau(\lambda)$, which is a measure of the linear delay between two time-series is defined to be proportional to the derivative of the phase spectrum:

$$
2 \pi \times \tau(\lambda)=-(d / d \lambda) \phi(\lambda)
$$

Thus, for the frequency band of interest, we can estimate a linear phase-delay as the average slope of the phase-spectrum within the band of interest. In summary, we extract two real-valued functions, coherence, and phase-delay, from the magnitude and phase components of coherency. The latter provides an index of which signal leads or lags the other in time.

Identifying seed voxels. To isolate seed voxels in the fusiform gyrus selectively engaged during face processing, BOLD responses to blocks of face and scene stimuli in the face/scene localizer task were modeled with $16 \mathrm{~s}$ regressors. These epochs were then convolved with a canonical HRF and effects were estimated under the assumptions of the general GLM analysis. Following parameter estimation, a linear contrast of face stimuli vs. scene stimuli was computed for each subject in normalized space. Voxels showing significantly greater activation for face stimuli than scenes in each participant's fusiform gyrus were then utilized as subject-specific seeds in the FFA coherence analysis.

Other seed regions of interest in prefrontal and parietal cortices were identified at the group level based upon whole-brain map-wise statistical analyses. Seed voxels of interest in the PFC were extracted from the task-common and task-selective group maps based upon anatomical boundaries of the superior, middle, and inferior frontal gyri in the PFC. This combined functional/anatomical approach was also used to isolate voxels in bilateral IPS and precuneus. Suprathreshold voxels within a $7 \mathrm{~mm}$ sphere of the peak voxel in these regions were included in the ROI masks. These group-level seed voxels were then passed on for second order coherence analyses on each participant's normalized functional data.

Generating time-series and coherence maps. Time-series for each voxel in the brain were separated into continuous timeseries by concatenating the two runs of each condition together. The two 378 segments of each condition were mean-centered, tapered with a four-point split-cosine bell function to limit spectral leakage due to edge effects in between the two independent blocks, and then concatenated together. We estimated the band-averaged $(0-0.15 \mathrm{~Hz})$ condition-specific coherence of the seed region with all other voxels across the brain using Welch's averaged periodogram method implemented in house scripts in Matlab (http://www.mathworks.com) (Oppenheim and Schafer, 1989). We chose this frequency band because it ecompasses the peak of the hemodynamic transfer function. In other words, henodynamic frequencies that contain signal which is mediated by changes in neural activity. As delineated above, a band-average coherence value of 1 would indicate that the time-series are perfectly related by a linear transform across all frequencies in the band, and a coherence of 0 indicates the complete lack of this relationship. Coherence magnitude and phase delay maps for each condition were then constructed based upon the voxel-wise coherence magnitude and phase values, respectively.

Contrasting coherence across encoding and retrieval conditions. To investigate functional connectivity with seed regions across encoding and retrieval, we subtracted the Guassian-normalized coherence maps for encoding and retrieval. The normalization transformation, accomplished using the arc-hyperbolic tangent function, allowed us to apply a parametric random-effects group analysis on the difference maps (Rosenberg et al., 1989). A one sample $t$-test was performed across subjects at a map-wise level with a significance value set at $p<0.005(k=10)$.

Temporal analysis: coherence phase delays. To characterize the temporal relationships between different interacting regions in the mnemonic networks, we investigated multiple phase measures between coherent sets of voxels. For each coherence seed (e.g., the FFA), regions across the brain found to be significantly coherent at the group-level (following group averaging) were isolated as "search space" ROIs to extract phase delay measures from each participant's whole-brain phase delay maps. These ROIs were isolated by taking a $10 \mathrm{~mm}$ sphere around the peak coherence voxel at the group level. These group-level search ROIs were used as masks on each participant's normalized coherence maps to extract the subset of voxels within that group ROI that showed significant coherence for that participant. These individual subject ROIs were then used to extract phase delay measures from each ROI for each participant. This analysis ensured that, at the individual subject level, phase values were only being extracted for voxels that had significant coherence with the seed of interest. Two participants were excluded from this temporal analysis due to the absence of coherent voxels in a number of the group search-space ROIs shown to be coherent at the group level with the FFA. Additionally, given the small number of voxels in the bilateral hippocampus significantly coherent at the group level, the voxels comprising the hippocampus search-space ROI were pooled from both hemispheres.

\section{RESULTS \\ BEHAVIORAL DATA}

Despite the large number of memoranda during encoding, participants performed significantly greater than chance (mean hit 
rate: 0.65 ; S.D. $=0.07)$ during the recognition test blocks with a mean reaction time of $1509 \mathrm{~ms}$. Three subjects were excluded from the behavioral analysis due to technical problems in collecting button-press responses. Given that coherence measures are summary values derived from analysis of the entire conditionspecific time-series, it is not possible to examine the link between functional connectivity and behavior (e.g., subsequent memory effects) on a trial-by-trial basis. While analyses examining the link between coherence values and overall recognition accuracy are possible, these analyses were inconclusive given this lack of sensitivity to trial-wise links between coherence measures and behavior outcome and due to limited variability in behavioral performance on the task across participants.

\section{IMAGING DATA: UNIVARIATE ANALYSES \\ Task common regions (encoding and retrieval)}

To isolate task-common regions, we formed a map of areas significantly active in both encoding and retrieval conditions (see Figure 1). Regions that overlapped across both mnemonic operations included a set of unimodal and multimodal regions known to be involved in bottom-up sensory processing of visual and, more particularly, face representations: bilateral calcarine cortex, right thalamus, fusiform gyrus, and lingual gyrus. Other regions activated under both mnemonic operations included the left putamen, right inferior frontal gyrus (IFG), left anterior IPS (aIPS), right aIPS, bilateral precuneus, bilateral anterior cingulate, bilateral supplementary motor area (SMA), left primary motor cortex, and left parahippocampal gyrus. At a lower statistical threshold ( $p<0.005$, uncorrected), right middle frontal gyrus (MFG), right caudate, bilateral MTL (with voxels located in both hippocampus and parahippocampus), and bilateral insula were also engaged during both encoding and retrieval.

\section{Task-selective regions (encoding)}

A linear contrast of encoding and retrieval maps at the group level (Figure 1) revealed greater activation during encoding in the left MFG $(-25,31,37)$. Additional encoding-selective regions included the left superior parietal lobule $(-36,-68,54)$, left parahippocampus $(-16,33,-14)$ and right $\operatorname{SMA}(6,-21,57)$.

\section{Task-selective regions (retrieval)}

A linear contrast of encoding and retrieval maps at the group level (Figure 1) revealed greater activation during retrieval within right pIPS $(32,-62,41)$, and right precuneus $(14,-63,41)$. Additional

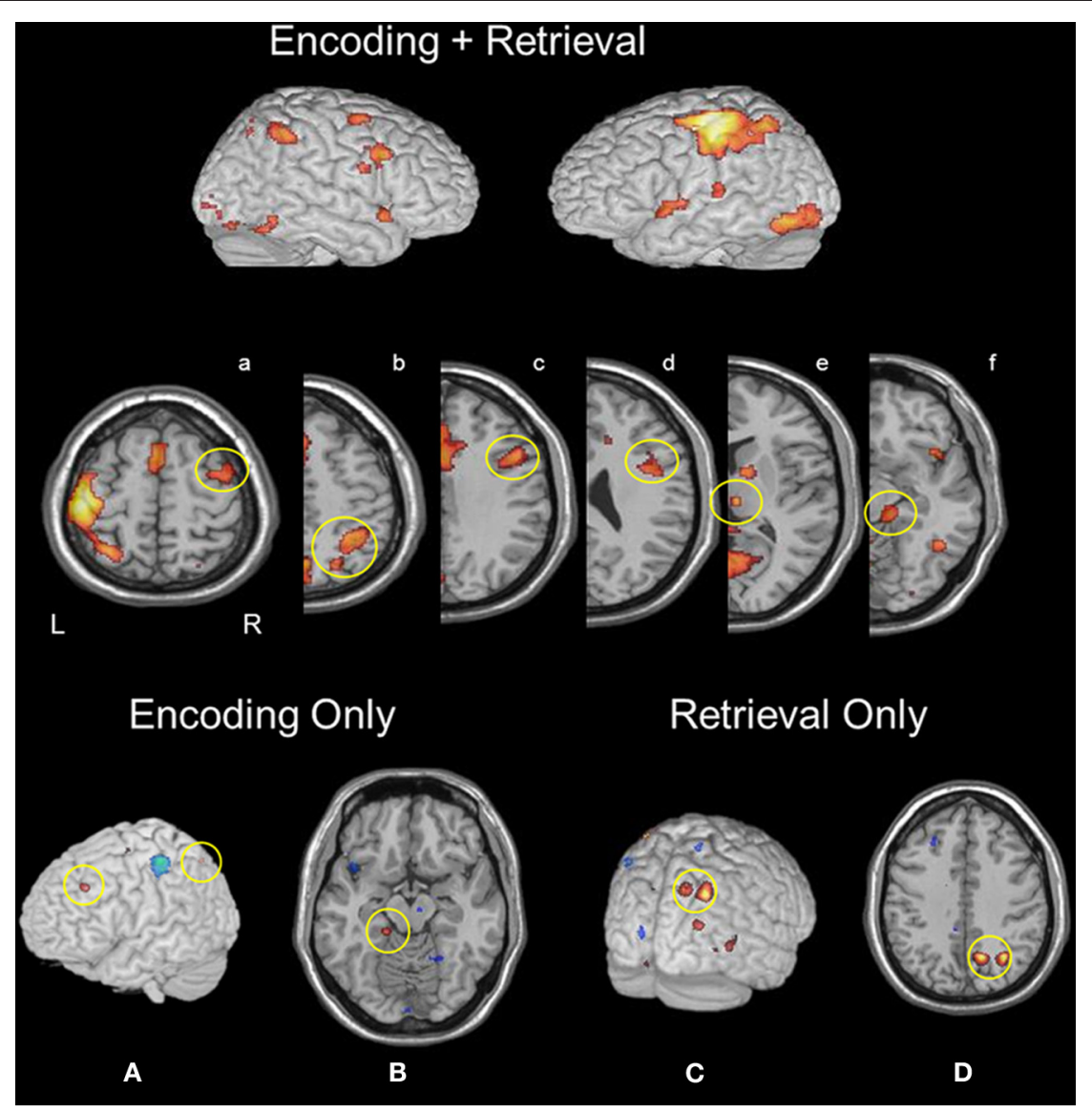

FIGURE 1 | Regions active during both memory encoding and retrieval: (a) rMFG, (b) right alPS and pIPS, (c and d) rIFG, (e) right thalamus, and (f) right parahippocampus. Encoding selective activity was identified in (A) left MFG and IPS, and (B) left parahippocampus. Retrieval selective activity was found in (C) right precuneus and (D) right pIPS. 
retrieval-selective regions were found in left post-central gyrus $(-27,36,66)$ and right putamen $(28,11,0)$. At a lower threshold ( $p<0.01$, uncorrected), right IFG activation was found.

\section{IMAGING DATA: COHERENCE ANALYSES}

\section{Fusiform gyrus coherence maps during both memory encoding and retrieval}

Subject-specific voxels within fusiform gyrus (presumed to be involved in face processing) were used as a seed in coherence analyses aimed at assessing functional connectivity during encoding and retrieval (see Materials and Methods and Figure 2). Given the role of the fusiform gyrus in perceptual processes engaged by both conditions of the face memory task, this analysis was aimed at assessing the extent to which the network of areas interacting with bottom-up regions across encoding and retrieval are distinct. During both encoding and retrieval, the right fusiform gyrus exhibited significant connectivity with bilateral MFG, bilateral IFG, bilateral SMA, left primary motor cortex, bilateral pIPS, bilateral precuneus, bilateral thalamus, bilateral calcarine cortex, left fusiform gyrus, bilateral medial occipital cortex, and bilateral hippocampus.

\section{Fusiform gyrus coherence maps specific to memory encoding or retrieval}

While the brain networks interacting with the fusiform gyrus were strikingly similar across mnemonic operations, a contrast of condition-specific coherence maps revealed operation-specific changes in coherence magnitude across regions. During encoding (relative to retrieval), the fusiform gyrus exhibited significant coherence with the right superior frontal gyrus (SFG, BA10). During retrieval (relative to encoding), the fusiform gyrus was

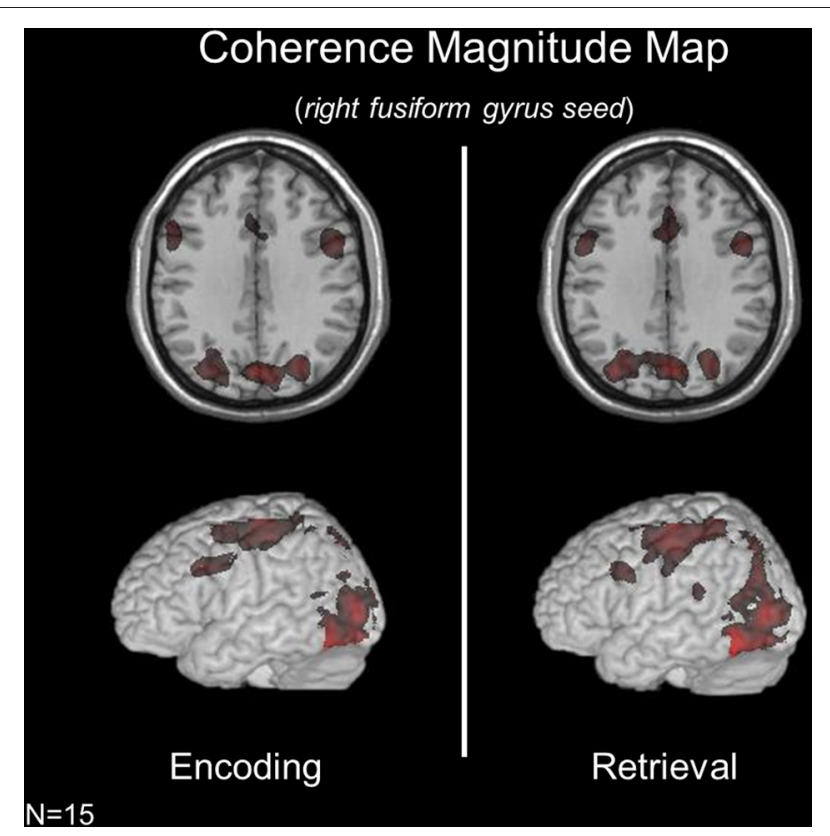

FIGURE 2 | Coherence magnitude analyses using a right fusiform gyrus seed across memory conditions revealed highly overlapping networks during encoding and retrieval. significantly more coupled with the left MFG and left precuneus. Importantly, these changes in the strength of connectivity between regions are in the presence of identical bottom-up sensory input.

\section{Coherence phase relationships across task conditions}

Though face memory networks are notably overlapping across encoding and retrieval, coherence magnitude measures cannot capture temporal relationships within these networks. As a result, networks that spatially overlap can exhibit different network dynamics - and thereby different network contributions to behavior (McIntosh et al., 2004). Thus, we extracted mean phase delay measures from these encoding and retrieval coherence maps in ROIs in the PFC, pIPS, and bilateral hippocampus. Due to a limited number of voxels coherent in the hippocampus, we performed all analyses with a joint ROI comprised of voxels from both hemispheres.

To assess regional differences in temporal relationships between the fusiform gyrus and ROIs in the PFC, parietal cortex and hippocampus across conditions we performed a Region (right MFG, left MFG, right IFG, left IFG, right aIPS, left aIPS, hippocampus) by Condition (Encoding vs. Retrieval) ANOVA on coherence phase measures. A significant Region X Condition interaction $(p<0.04)$ was found suggesting different temporal relationships between the ROIs across processing states. Planned comparisons revealed that during both encoding and retrievalbilateral IFG and IPS exhibited earlier activity than fusiform gyrus activity (see Figure 3). In addition, during encoding, there was no difference between phase estimates in the IFG and IPS, however, during retrieval, IFG activity was significantly earlier than IPS activity. During both encoding and retrieval, hippocampal activity lagged significantly behind fusiform activity.

\section{DISCUSSION}

The contributions of PFC and MTL regions to subcomponent memory operations have been supported through numerous experiments highlighting the engagement of these regions during the formation and retrieval of representations in both WM

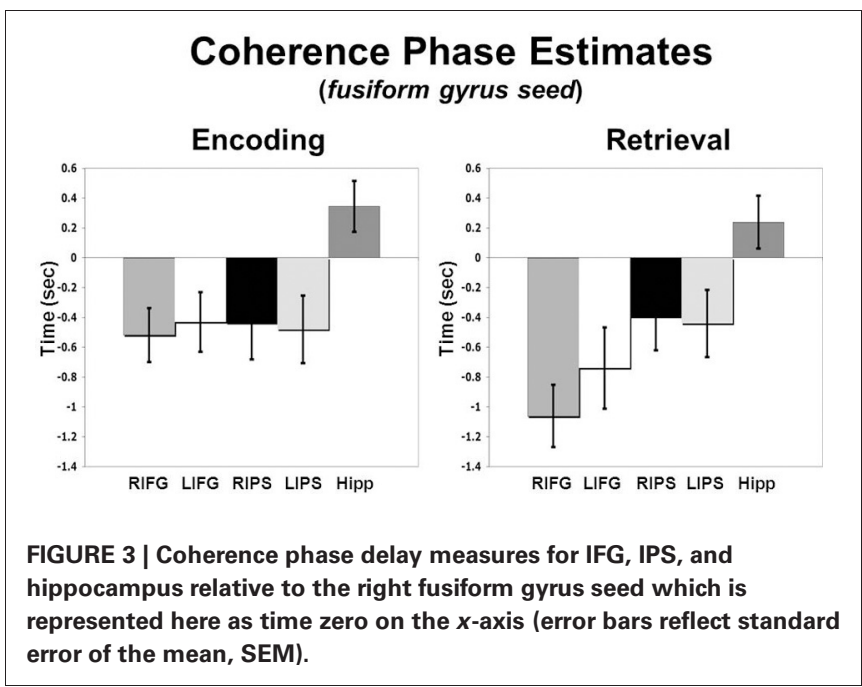


and LTM. More recently, empirical evidence has emerged highlighting a role of PPC in memory function. In our experiment, all three regions were engaged during the encoding and retrieval of episodic memories for faces. It is proposed that mnemonic control signals emanating from PFC and PPC can modify perceptual processing of memoranda and integrate with MTL regions to strengthen and access internal representations of this information. In this experiment, we utilized coherence analyses of fMRI data to test this notion and further characterize the nature of the interactions of these critical regions during encoding and retrieval of episodic memories of faces. We demonstrated significant functional connectivity between the FFA and PFC, PPC and hippocampus during both memory operations. Moreover, we found that PFC and PPC are engaged earlier than FFA during both operations-placing them in a temporal window to guide memory function in a top-down manner. In contrast, hippocampal activity lagged behind both PFC, PPC, and FFA during both encoding and retrieval operations. The potential functional contributions of these interactions are discussed below.

\section{FUNCTIONAL CONNECTIVITY AMONG BRAIN REGIONS DURING MEMORY FUNCTION}

Univariate analyses demonstrated several cortical regions that were engaged during both encoding and retrieval conditions which included primary and visual association cortex, as well multimodal regions within parietal and frontal cortex. Despite known anatomical connections (for a review, see Goldman-Rakic, 1988), univariate analyses of imaging data cannot provide information about connectivity or influences between regions. Thus, we utilized complementary multivariate statistics to provide a systems-level understanding of the distributed neural interactions underlying mnemonic function. Measuring coherence, we delineated networks of brain regions functionally connected with FFA during episodic memory encoding and retrieval. Interestingly, some of the regions that exhibited functional connectivity with FFA was not identified in the univariate analyses, highlighting the added value of multivariate approaches. Coherency analyses demonstrated that the FFA was functionally connected with PFC, parietal cortex, and MTL during both encoding and retrieval.

Involvement of the PFC in multiple processes for encoding and retrieving of object (and specifically face) LTM representations is consistent with its known anatomical connectivity. For example, the IFG has reciprocal connections with multiple subregions of the inferior temporal cortex (Webster et al., 1994; Petrides and Pandya, 1999). Specifically, the IFG (BA 44/45) engaged across conditions in our task have been linked to the selection of item or object-based visual LTM representations (e.g., Petrides, 2002; Ranganath, 2006). A more dorsal region of lateral PFC (i.e., MFG), which was also engaged across both conditions, is proposed to be critical for processing the relations between LTM representations currently active in memory (Davachi and Wagner, 2002; Blumenfeld and Ranganath, 2006). Although our task did not directly vary relational processing during encoding, MFG activation may reflect strategic relational processes required for binding specific features of each face stimulus to distinguish it from interfering representations within the large set of memoranda. These strategies may have been employed given that the face stimuli in our task had and other distinguishing characteristics were removed - resulting in a high overlap in similarity across stimuli.

During LTM encoding and retrieval operations, the FFA was coherent with the PPC in addition to PFC, again, consistent with known anatomical connectivity. Multiple parietal subregions exhibit resting state correlations with the hippocampus (Vincent et al., 2006) and superior parietal cortex exhibits anatomical and functional connectivity with sensory areas in lateral inferotemporal cortex (Takahashi et al., 2008). This data supports a role of PPC in top-down signaling in episodic memory, consistent with a growing literature showing it is engaged during different episodic memory demands (e.g., Konishi et al., 2000; Dobbins et al., 2003; Wheeler and Buckner, 2004; Buchsbaum et al., 2011) as well as contributions to mental imagery - which requires reactivation of visual LTM representations in the face of no bottom-up sensory input (Mechelli et al., 2004). The degree to which these signals are causal contributors to behavior is unclear since parietal lesions do not impair recognition memory (Haramati et al., 2008; for a review of this “posterior parietal paradox," see Schoo et al., 2010).

Subregions of the MTL, including the hippocampal formation, parahippocampus, and perirhinal cortex have been implicated in a number of processes that underlie episodic LTM. The specific functional role of each subregion remains remains hotly debated. While disentangling contributions of specific MTL subregions is best suited for high-resolution fMRI studies, our findings of FFA/MTL connectivity findings may shed some light on the functional contributions of substructures within the MTL. Models of MTL function highlight a specific role for the hippocampal formation in binding representations of the relations between items and stimuli in the environment (Giovanello et al., 2004; Ryan and Cohen, 2004; Davachi, 2006). On the other hand, evidence has linked parahippocampal recruitment with the encoding and perception of spatial relations and perirhinal cortex with the encoding of individual item representations (for a review, see Davachi, 2006). In our experiment, we would predict selective engagement of perirhinal cortex during encoding trials given the lack of any overt relational component to the task. However, we found hippocampal activity (and robust FFA-hippocampal connectivity) during both memory conditions. These patterns seemingly argue against a selective involvement of the hippocampus in the encoding of relational information. It is possible, however, given the absence of any controlled encoding, that participants may have adopted some level of a relational strategy during the course of their intentional memorization. Recent evidence has shown hippocampal involvement in the formation of face/name relations (Sperling et al., 2003; Kirwan and Stark, 2004) and, along these lines, in a post-hoc assessment subjects often reported using names or verbal descriptive cues as a strategy to aid in subsequent recognition.

Neurophysiology and neuroimaging research has provided a wealth of suggestive evidence showing PFC and parietal involvement in tasks presumed to require top-down modulatory input from higher order areas to extrastriate visual regions (for a review specific to the PFC, see Miller and D'Esposito, 2005). Recently, other investigations have employed multivariate statistical methods and have found PFC-visual association cortex 
coupling during WM encoding (Gazzaley et al., 2007) and WM maintenance (Gazzaley et al., 2004). Our findings suggest that LTM encoding and retrieval are mediated—at least in part-by similar coupling.

\section{COHERENCE PHASE MEASURES DURING LTM ENCODING AND RETRIEVAL}

While coherence magnitude measures establish coupling between multimodal association cortex and the FFA, they fail to directly assess the temporal dynamics of these interactions. PFC-FFA coupling during encoding, for example, could reflect that PFC receives feed-forward signals from the FFA allowing it to monitor and manipulate sensory representations (Petrides et al., 2002), or accumulate sensory evidence in favor of a perceptual or mnemonic decision (Kim and Shadlen, 1999). Alternatively, this coupling could reflect a top-down signal emanating from PFC to bias sensory processing in favor of task demands (e.g., Moore and Armstrong, 2003). These two fundamentally different interactions (amongst other possibilities) are equally consistent with our functional connectivity results but coherence phase estimates may provide additional evidence regarding the dynamics of this connectivity.

We used coherence phase estimates to examine the timing relationships between FFA, PFC, PPC and hippocampus activity across encoding and retrieval conditions. First, we found that PFC and PPC led the FFA in time during both encoding and retrieval operations. This temporal pattern places both the PFC and PPC in a temporal window to modify bottom-up processing in the FFA in a top-down manner. While it remains difficult to map BOLD latency measures directly back onto underlying neurophysiological patterns (a challenge referred to as the "Hemodynamic Inverse Problem," Buckner, 2003), we believe that the interpretation of these findings in the context of existing neurophysiology evidence is informative. As an important note-several of the phase relationships we observed were on a longer time scale than typically recorded in the rapid sampling methods of ERP, magnetoencephalography (MEG), and single-unit neurophysiology. Thus, it is not clear whether these time delays are related to delays caused by the HRF or if there may be psychological explanations (e.g., Schacter et al., 1997; Druzgal and D'Esposito, 2003).

\section{Top-down interactions with the FFA}

Our findings suggest that there was rapid engagement of multimodal association regions - even before bottom-up processing of face stimuli by the FFA. This is consistent with previous evidence showing that PFC becomes engaged rapidly and within a time window to sculpt sensory processing in a top-down manner (e.g., Druzgal and D'Esposito, 2003). For example, MEG data (Bar et al., 2006) implicated orbitofrontal regions in early top-down facilitation of object recognition. PFC signals at $130 \mathrm{~ms}$-led inferotemporal cortex by $50 \mathrm{~ms}$. These early frontal effects were hypothesized to be driven by rapid (low spatial-frequency) visual signals that allow the PFC to generate predictions about the nature of a visual stimulus and initiate top-down signals to corresponding extrastriate regions to bolster bottom-up processing in those regions. Other physiology studies have demonstrated that PFC neuronal activity reflects the mnemonic status of a stimulus before neurons in IT cortex. While the face-selective ERP component-which is hypothesized to reflect coherent neural synchronization in the FFA-has been shown to peak at around $170 \mathrm{~ms}$ following stimulus onset, PFC neurons have been shown to reflect target sensitivity as early as $140 \mathrm{~ms}$ following stimulus onset (Thompson et al., 1997; Rainer et al., 1998). This is significantly before the presumed time of maximal FFA response and also earlier than target sensitive firing in IT neurons during visual search ( $\sim 180$ ms, Chelazzi et al., 1993).

A second finding was that, relative to FFA responses, PFC activity (i.e., right IFG) was significantly earlier at retrieval compared to encoding. This context-dependent shift in timing reflects changing network dynamics in the presence of identical bottom-up processing. Since it is unlikely that the PFC and FFA hemodynamic response changes across encoding vs. retrieval, this condition-specific change in phase is likely neural in origin. An unresolved question is whether this early PFC response during retrieval is stimulus-driven or a pre-stimulus anticipatory signal. The PFC has been shown to exhibit early preparatory signals when establishing a "retrieval mode" in anticipation of test probes, however, such a response is typically found in more anterior PFC regions (BA10; Cabeza et al., 2002; Ranganath et al., 2003). Nevertheless, our coherence findings suggest a role of ventrolateral PFC in early control operations during episodic LTM recognition tasks.

\section{Frontal-parietal interactions}

We also found that PFC activity preceded PPC activity only during retrieval. PFC and PPC often exhibit similar response patterns in tasks which engage WM operations, visual search, orienting of attention, and the maintenance of a task set. While some recent experiments have devised novel cognitive manipulations and used standard univariate analyses to dissociate processing in these areas (e.g., Champod and Petrides, 2007), differences in functional and effective connectivity between the PFC and PPC offer new information to differentiate the respective roles of these regions. Previous work showing direct evidence of functional connectivity between PFC and PPC comes from single-unit recordings in the PFC following cryogenic deactivation of the parietal cortex. Quintana et al. (1989) showed that parietal cooling led to alterations in PFC signaling at multiple stages of a WM task.

The earlier activity of the PFC relative to parietal cortex in the current investigation at retrieval could shed important light on the different functional roles of these regions in top-down control operations. For example, a similar pattern emerged in a monkey study examining the temporal relationship between the PFC and parietal cortex during memory-guided attention (Buschmman and Miller, 2007). During a pop-out attention condition elicited by a distinguishable visual stimulus, spiking activity began earlier in parietal neurons than PFC neurons. In contrast, during a visual search condition, when attention must be deployed based upon memory for a particular target of interest, this relative order of activity onset reversed. Relative to our findings, early PFC influences of parietal cortex at retrieval could guide attentional allocation to stimulus dimensions most relevant for making memory-guided recognition decisions at retrieval. This could 
lead to increased processing of the dimensions of each probe face that differentiate it from the rest of the memoranda and test items to support familiarity or recollection-based memory decisions.

\section{Hippocampal interactions}

While the hippocampus is known to have reciprocal connectivity with inferior temporal cortex (Yoshida et al., 2003), few studies have explored the temporal characteristics of this coupling simultaneously across regions. In the current study, significant coherence between bilateral hippocampus and the FFA were found during both LTM encoding and retrieval. Across both memory conditions, hippocampal activity lagged behind PFC, PPC, and FFA activity. Single-unit physiology work in non-human primates has shown that hippocampal neurons exhibit initial responses to object stimuli $\sim 210 \mathrm{~ms}$ post-stimulus (Eifuku et al., 1995) but these latencies vary across different stimulus categories (e.g., Rolls et al., 1993). While these timing profiles suggest that hippocampal responses lag behind mean population responses in IT neurons (and those thought reflecting high-level face processing more specifically), recent work has further investigated these physiological patterns in human patients. For example, Mormann et al. (2008) demonstrated that while hippocampal neurons exhibit stimulus selectivity to visual objects, these neurons responded significantly later (i.e., $394 \mathrm{~ms}$ ) than other MTL subregions. These findings suggest differences across human and animal models and but are generally consistent with our fMRI data indicating later hippocampal activity during face encoding and recognition decisions.

It is important to consider our coherence findings (magnitude and phase) within the context of current models of the role of the hippocampus and other subregions of the MTL (e.g., parahippocampus) in episodic LTM. In our study, although the hippocampus exhibited significant connectivity with the FFA across both mnemonic conditions, other MTL regions were also significantly active, as demonstrated in the univariate analyses of the imaging data. Thus, it is likely the parahippocampus and perirhinal cortex were also involved in our task, which is consistent with previous findings indicating an important role of perirhinal cortex in item recognition (e.g., Yonelinas, 2002; Brown et al., 2010) and the parahippocampus in visual object processing and

\section{REFERENCES}

Allen, E. A., Pasley, B. N., Duong, T., and Freeman, R. D. (2007). Transcranial magnetic stimulation elicits coupled neural and hemodynamic consequences. Science 317, 1918-1921.

Antzoulatos, E. G., and Miller, E. K. (2011). Differences between neural activity in prefrontal cortex and striatum during learning of novel abstract categories. Neuron 71, 243-249.

Bar, M., Kassam, K. S., Ghuman, A. S., Boshyan, J., Schmid, A. M., Dale, A. M., Hamalainen, M. S., Marinkovic, K., Schacter, D. L., Rosen, B. R., and Halgren, E. (2006). Top-down facilitation of visual recognition. Proc. Natl. Acad. Sci. U.S.A. 103, 449-454.

Blumenfeld, R. S., and Ranganath, C. (2006). Dorsolateral prefrontal cortex promotes long-term memory formation through its role in working memory organization. J. Neurosci. 26, 916-925.

Brown, M. W., Warburton, E. C., and Aggleton, J. P. (2010). Recognition memory: material, processes, and substrates. Hippocampus 20, 1228-1244.

Buchsbaum, B. R., Ye, D., and D'Esposito, M. (2011). Recency effects in the inferior parietal lob during verbal recognition memory. the representation of the spatio-temporal context in which items were encountered (e.g., Ranganath, 2010). The specific functional importance of hippocampal-fusiform interactions across conditions remains unclear. While most current models (e.g., Ranganath, 2010) implicate the hippocampus in binding items with context or in representing higher level relational information about items (e.g., Eichenbaum and Cohen, 2001), the current task did not explicitly tax relational processing or source-based decisions at retrieval. While further experiments with high spatial resolution fMRI will be necessary to address the contribution of different MTL subsystems to these basic operations, the coupling and latency data present here are consistent with previous findings suggesting a late role of the hippocampus in recognition decisions.

\section{CONCLUSIONS}

In the current experiment, coherence analyses of fMRI data revealed overlapping networks interacting with the FFA during the encoding and retrieval of face stimuli. Additionally, coherence phase delay estimates reflecting the temporal dynamics of these networks revealed that PFC and PPC likely influences early visual areas during both LTM encoding and retrieval. This early PFC activity was even more pronounced at retrieval, preceding both IPS and FFA while the hippocampus lagged behind. Further work exploring these spatio-temporal interactions across memory operations and domains of memoranda will provide critical information to determine the functional role of network interactions to behavior. While fMRI remains a correlational technique and direct tests of these interactions will require future work combining physiology measures with functional deactivation through lesion or transcranial magnetic stimulation (Allen et al., 2007), coherence analyses offer a unique methodological strategy for tracking bottom-up and top-down interactions across regions.

\section{ACKNOWLEDGMENTS}

The authors thank Sven Collette for assistance in data analysis and Felice Sun, Lee Miller, Thomas Lauritzen, and Jesse Rissman for valuable conversations. Work was supported by National Institute of Health, Grants MH63901 and NS40813.
Front. Hum. Neurosci. 5:59. doi: 10.3389/fnhum.2011.00059

Buckner, R. L. (2003). The hemodynamic inverse problem: making inferences about neural activity from measured fMRI signals. Proc. Natl. Acad. Sci. U.S.A. 100, 2177-2179.

Buschmman, T. J., and Miller, E. K. (2007). Top-down versus bottomup control of attention in the prefrontal and posterior parietal cortices. Science 315, 1860-1862.

Cabeza, R., Dolcos, F., Prince, S. E., Rice, H. J., Weissman, D. H., and Nyberg, L. (2002). Attentionrelated activity during episodic memory retrieval: a cross-function
fMRI study. Neuropsychologia 41 390-399.

Cabeza, R., Locantore, J. K. and Anderson, N. D. (2003). Lateralization of prefrontal activity during episodic memory retrieval: evidence for the production-monitoring hypothesis. J. Cogn. Neurosci. 15, 249-259.

Champod, A. S., and Petrides, M. (2007). Dissociable roles of the posterior parietal and the prefrontal cortex in manipulation and monitoring processes. Proc. Natl. Acad. Sci. U.S.A. 104, 14837-14842.

Chelazzi, L., Miller, E. K., Duncan, J., and Desimone, R. (1993). A 
neural basis for visual search in inferior temporal cortex. Nature 363, 345-347.

Chun, M. M., and Turk-Browne, N. B. (2007). Interactions between attention and memory. Curr. Opin. Neurobiol. 17, 177-184.

Davachi, L. (2006). Item, context and relational episodic encoding in humans. Curr. Opin. Neurobiol. 16, 693-700.

Davachi, L., and Wagner, A. D. (2002). Hippocampal contributions to episodic encoding: insights from relational and item-based learning. J. Neurophysiol. 88, 982-990.

Dobbins, I. G., Rice, H. J., Wagner, A. D., and Schacther, D. L. (2003). Memory orientation and success: separable neurocognitive components underlying episodic recognition. Neuropsychologia 41, 318-333.

Druzgal, T. J., and D’Esposito, M. (2003). Dissecting contributions of prefrontal cortex and fusiform face area to face working memory. $\mathrm{J}$. Cogn. Neurosci. 15, 771-784.

Eichenbaum, H., and Cohen, N. J. (2001). From Conditioning to Conscious Recollection: Memory Systems of the Brain. New York, NY: Oxford University Press.

Eifuku, S., Nishijo, H., Kita, T., and Ono, T. (1995). Neuronal activity in the primate hippocampal formation during a conditional association task based on the subject's location. J. Neurosci. 15, 4952-4969.

Garrido, M. I., Kilner, J. M., Kiebel, S. J., and Friston, K. J. (2007). Evoked brain responses are generated by feedback loops. Proc. Natl. Acad. Sci. U.S.A. 104, 20961-20966.

Gazzaley, A., Cooney, J. W., Rissman, J., and D'Esposito, M. (2005). Topdown suppression deficit underlies working memory impairment in normal aging. Nat. Neurosci. 8, 1298-1300.

Gazzaley, A., Rissman, J., Cooney, J., Rutman, A., Seibert, T., Clapp, W., and D'Esposito, M. (2007). Functional interactions between prefrontal and visual association cortex contribute to top-down modulation of visual processing. Cereb. Cortex (Suppl 1), i125-i135.

Gazzaley, A., Rissman, J., and D'Esposito, M. (2004). Functional connectivity during working memory maintenance. Cogn. Affect. Behav. Neurosci. 4, 580-599.

Giovanello, K. S., Schnyer, D. M., and Verfaellie, M. (2004). A critical role for the anterior hippocampus in relational memory: evidence from an fMRI study comparing associative and item recognition. Hippocampus 14, 5-8.

Goldman-Rakic, P. S. (1988). Topography of cognition: parallel distributed networks in primate association cortex. Annu. Rev. Neurosci. 11, 137-156.

Haramati, S., Soroker, N., Dudai, Y., and Levy, D. A. (2008). The posterior parietal cortex in recognition memory: a neuropsychological study. Neuropsychologia 46, 1756-1766.

Hutchinson, J. B., Uncapher, M. R., and Wagner, A. D. (2009). Posterior parietal cortex and episodic retrieval: convergent and divergent effects of attention and memory. Learn. Mem. 16, 343-356.

Kim, J. N., and Shadlen, M. N. (1999). Neural correlates of a decision in the dorsolateral prefrontal cortex of the macaque. Nat. Neurosci. 2, 176-185.

Kirwan, C. B., and Stark, C. E. (2004). Medial temporal lobe activation during encoding and retrieval of novel face-name pairs. Hippocampus 14, 919-930.

Knudsen, E. I. (2007). Fundamental components of attention. Аnnu. Rev. Neurosci. 30, 57-78.

Konishi, S., Wheeler, M. E., Donaldson, D. I., and Buckner, R. L. (2000). Neural correlates of episodic retrieval success. Neuroimage 12, 276-286.

Kveraga, K., Bosyhan, J., and Bar, M. (2007). Magnocellular projections as the trigger of top-down facilitation in recognition. J. Neurosci. 27, 13232-13240.

McIntosh, A. R., Chau, W., and Protzner, A. B. (2004). Spatiotemporal analysis of eventrelated fMRI data using partial least squares. Neuroimage 23, 764-775.

Mechelli, A., Price, C. J., Friston, K. J., and Ishai, A. (2004). Where bottom-up meets top-down: neuronal interactions during perception and imagery. Cereb. Cortex 14, 1256-1265.

Mesulam, M. M. (1998). From sensation to cognition. Brain 121, 1013-1052.

Miller, B. T., and D'Esposito, M. (2005). Searching for 'the top' in top-down control. Neuron 48, 535-538.

Miller, E. K., and Cohen, J. D. (2001). An integrative theory of prefrontal cortex function. Annu. Rev. Neurosci. 24, 167-202.

Moore, T., and Armstrong, K. M. (2003). Selective gating of visual signals by microstimulation of frontal cortex. Nature 421, 370-373.

Mormann, F., Kornblith, S., Quiroga, R. Q., Kraskov, A., Cerf, M., Fried,
I., and Koch, C. (2008). Latency and selectivity of single neurons indicate hierarchical processing in the human medial temporal lobe. J. Neurosci. 28, 8865-8872.

Oppenheim, A. V., and Schafer, R. W. (1989). Discrete-Time Signal Processing. Englewood Cliffs, NJ: Prentice Hall.

Petrides, M. (2002). The midventrolateral prefrontal cortex and active mnemonic retrieval. Neurobiol. Learn. Mem. 78, 528-538.

Petrides, M., Alivisatos, B., and Frey, S. (2002). Differential activation of the human orbital, mid-ventrolateral, and mid-dorsolateral prefrontal cortex during the processing of visual stimuli. Proc. Natl. Acad. Sci. U.S.A. 99, 5649-5654.

Petrides, M., and Pandya, D. N. (1999). Dorsolateral prefrontal cortex: comparative cytoarchitectonic analysis in the human and the macaque brain and corticocortical connection patterns. Eur. J. Neurosci. 11, 1011-1036.

Quintana, J., Fuster, J. M., and Yajeya, J. (1989). Effects of cooling parietal cortex on prefrontal units in delay tasks. Brain Res. 503, 100-110.

Rainer, G., Asaad, W. F., and Miller, E. K. (1998). Memory fields of neurons in the primate prefrontal cortex. Proc. Natl. Acad. Sci. U.S.A. 95, 15008-15013.

Ranganath, C. (2006). Working memory for visual objects: complementary roles of inferior temporal, medial temporal, and prefrontal cortex. Neuroscience 139, 277-289.

Ranganath, C. (2010). A unified framework for the functional organization of the medial temporal lobes and the phenomenology of episodic memory. Hippocampus 20, 1263-1290.

Ranganath, C., and D'Esposito, M. (2005). Directing the mind's eye prefrontal inferior and medial temporal mechanisms for visual working memory. Curr. Opin. Neurobiol. $15,175-182$.

Ranganath, C., Heller, A., Cohen, M. X., Brozinsky, C. J., and Rissman, J. (2005). Functional connectivity with the hippocampus during successful memory formation. Hippocampus 15, 997-1005.

Ranganath, C., Johnson, M. K., and D'Esposito, M. (2003). Prefrontal activity associated with working memory and episodic long-term memory. Neuropsychologia 41, 378-389.

Rolls, E. T., Cahusac, P. M., Feigenbaum, J. D., and Miyashita, Y. (1993). Responses of single neurons in the hippocampus of the macaque related to recognition memory. Exp. Brain Res. 93, 299-306.

Rosenberg, J. R., Amjad, A. M., Breeze, P., Brillinger, D. R., and Halliday, D. M. (1989). The Fourier approach to the identification of functional coupling between neuronal spike trains. Prog. Biophys. Mol. Biol. 53, 1-31.

Ryan, J. D., and Cohen, N. J. (2004). Processing and short-term retention of relational information in amnesia. Neuropsychologia 42, 497-511.

Schacter, D. L., Buckner, R. L., Koutstaal, W., Dale, A. M., and Rosen, B. R. (1997). Late onset of anterior prefrontal activity during true and false recognition: an eventrelated fMRI study. Neuroimage 6 , 259-269.

Schoo, L. A., van Zandvoort, M. J., Biessels, G. J., Kappelle, L. J., Postma, A., and de Haan, E. H. (2010). The posterior parietal paradox: why do functional magnetic resonance imaging and lesion studies on episodic memory produce conflicting results? J. Neuropsychol. 5, 15-38.

Shimamura, A. P. (2000). The role of the prefrontal cortex in dynamic filtering. Psychobiology 28, 207-218.

Sperling, R., Chua, E., Cocchiarella, A., Rand-Giovannetti, E., Poldrack, R., Schacter, D. L., and Albert, M. (2003). Putting names to faces: successful encoding of associative memories activates the anterior hippocampal formation. Neuroimage 20, 1400-1410.

Summerfield, C., Greene, M., Wager, T., Egner, T., Hirsch, J., and Mangels, J. (2006). Neocortical connectivity during episodic memory formation. PLoS Biol. 4:e128. doi: 10.1371/journal.pbio.0040128

Sun, F. T., Miller, L. M., and D'Esposito, M. (2004). Measuring interregional functional connectivity using coherence and partial coherence analyses of fMRI data. Neuroimage 21, 647-658.

Sun, F. T., Miller, L. M., and D'Esposito, M. (2005). Measuring temporal dynamics of functional networks using phase spectrum of fMRI data. Neuroimage 28, 227-237.

Suzuki, W. A., and Amaral, D. G. (1994). Perirhinal and parahippocampal cortices of the macaque monkey: cortical afferents. J. Comp. Neurol. 350, 497-533.

Takahashi, E., Ohki, K., and Kim, D. S. (2007). Diffusion tensor studies dissociated two fronto-temporal pathways in the human memory system. Neuroimage 34, 827-838.

Takahashi, E., Ohki, K., and Kim, D. S. (2008). Dissociated pathways 
for successful memory retrieval from the human parietal cortex: anatomical and functional connectivity analyses. Cereb. Cortex 18, 1771-1778.

Thompson, K. G., Bichot, N. P., and Schall, J. D. (1997). Dissociation of visual discrimination from saccade programming in macaque frontal eye field. J. Neurophysiol. 77, 1046-1050.

Uncapher, M. R., and Wagner, A. D. (2009). Posterior parietal cortex and episodic encoding: insights from fMRI subsequent memory effects and dual-attention theory. Neurobiol. Learn. Mem. 91, 139-154.

Vincent, J. L., Snyder, A. Z., Fox, M. D., Shannon, B. J., Andrews, J.
R., Raichle, M. E., and Buckner, R. L. (2006). Coherent spontaneous activity identifies a hippocampal-parietal memory network. J. Neurophysiol. 96, 3517-3531.

Wang, L., Liu, X., Guise, K. G., Knight, R. T., Hajar, J., and Fan, J. (2010). Effective connectivity of the fronto-parietal network during attentional control. J. Cogn. Neurosci. 22, 543-553.

Webster, M. J., Bachevalier, J., and Ungerleider, L. G. (1994). Connections of inferior temporal areas TEO and TE with parietal and frontal cortex in macaque monkeys. Cereb. Cortex 4, 470-483.

Wheeler, M. E., and Buckner, R. L. (2004). Functional-ana- tomic correlates of remembering and knowing. Neuroimage 21, 1337-1349.

Yonelinas, A. P. (2002). The nature of recollection and familiarity: a review of 30 years of research. J. Mem. Lang. 46, 441-517.

Yoshida, M., Naya, Y., and Miyashita, Y. (2003). Anatomical organization of forward fiber projections from area TE to perirhinal neurons visual long-term memory in monkeys. Proc. Natl. Acad. Sci. U.S.A. 100, 4257-4262.

Conflict of Interest Statement: The authors declare that the research was conducted in the absence of any commercial or financial relationships that could be construed as a potential conflict of interest.

Received: 17 January 2012; accepted: 11 April 2012; published online: 01 May 2012.

Citation: Miller BT and D'Esposito $M$ (2012) Spatial and temporal dynamics of cortical networks engaged in memory encoding and retrieval. Front. Hum. Neurosci. 6:109. doi: 10.3389/fnhum. 2012.00109

Copyright (C) 2012 Miller and D'Esposito. This is an open-access article distributed under the terms of the Creative Commons Attribution Non Commercial License, which permits noncommercial use, distribution, and reproduction in other forums, provided the original authors and source are credited. 